\title{
A deep study of the high-energy transient sky
}

\author{
C. Guidorzi $i^{1,2,3}$ (D) F. Frontera1,2 . G. Ghirlanda 4,5 . G. Stratta ${ }^{2}$. C. G. Mundell 6 . \\ E. Virgilli ${ }^{1}$ - P. Rosati ${ }^{1,2,3}$ - E. Caroli ${ }^{2}$ - L. Amati ${ }^{2}$ - E. Pian ${ }^{2}$ - S. Kobayashi ${ }^{7}$. \\ G. Ghisellini ${ }^{4}$. C. Fryer ${ }^{8} \cdot$ M. Della Valle $9,10,11 \cdot$ R. Margutti $^{12} \cdot$ M. Marongiu ${ }^{1,11}$. \\ R. Martone ${ }^{1,11} \cdot$ R. Campana ${ }^{2}$. F. Fuschino ${ }^{2}$. C. Labanti ${ }^{2} \cdot$ M. Orlandini' ${ }^{2}$. \\ J. B. Stephen ${ }^{2}$. S. Brandt ${ }^{13} \cdot$ R. Curado da Silva ${ }^{14,15} \cdot$ P. Laurent ${ }^{16}$. \\ R. Mochkovitch ${ }^{17} \cdot$ E. Bozzo ${ }^{18} \cdot$ R. Ciolfi ${ }^{19,20}$. L. Burderi ${ }^{21} \cdot$ T. Di Salvo ${ }^{22}$
}

Received: 25 July 2020 / Accepted: 2 March 2021 / Published online: 23 April 2021

(C) The Author(s) 2021

\begin{abstract}
The coming decades will establish the exploration of the gravitational wave (GW) Universe over a broad frequency range by ground and space interferometers. Meanwhile, wide-field, high-cadence and sensitive surveys will span the electromagnetic spectrum from radio all the way up to $\mathrm{TeV}$, as well as the high-energy neutrino window. Among the numerous classes of transients, $\gamma$-ray bursts (GRBs) have direct links with most of the hot topics that will be addressed, such as the strong gravity regime, relativistic shocks, particle acceleration processes, equation of state of matter at nuclear density, and nucleosynthesis of heavy elements, just to mention a few. Other recently discovered classes of transients that are observed throughout cosmological distances include fast radio bursts (FRBs), fast blue optical transients (FBOTs), and other unidentified high-energy transients. Here we discuss how these topics can be addressed by a mission called ASTENA (Advanced Surveyor of Transient Events and Nuclear Astrophysics, see Frontera et al. 2021). Its payload combines two instruments: (i) an array of wide-field monitors with imaging, spectroscopic, and polarimetric capabilities (WFM-IS); (ii) a narrow field telescope (NFT) based on a Laue lens operating in the 50-600 keV range with unprecedented angular resolution, polarimetric capabilities, and sensitivity.
\end{abstract}

Keywords $\mathrm{X}-/ \gamma$-ray telescopes - Space mission concept .

ESA voyage 2050 • High-energy transient sky ·

Multi-messenger astrophysics $\cdot \mathrm{X}-/ \gamma$-ray polarimetry

C. Guidorzi

guidorzi@fe.infn.it

Extended author information available on the last page of the article. 


\section{Introduction}

The detection of gravitational and electromagnetic (EM) radiation from the binary neutron star (BNS) merger GW 170817 in 2017 heralded the beginning of the multi-messenger study of the transient sky. The GW Universe exploration over a broad frequency range by ground and space interferometers will reach the full maturity in the coming decades. Meanwhile, an armada of wide-field, high-cadence, and sensitive surveys are planned, spanning the EM spectrum from radio (SKA), through optical (the Rubin Observatory, previously known as the LSST), and up to X/ $\gamma$-ray (e.g THESEUS, accepted by ESA for a phase A study), and TeV (CTA), as well as particle-detectors such as those in the high-energy neutrino window (e.g., IceCubeGen2, KM3NeT). This towering effort will enable the study of events characterised by strong gravity effects, relativistic shocks, and particle acceleration processes over a broad mass, time, and distance scales. Not only will they address open issues of astrophysics, cosmology, and fundamental physics (e.g., formation of compact binaries, the equation of state of matter at nuclear densities, nucleosynthesis of heavy elements, cosmic-ray production, the nature of dark matter, and the cosmological parameters), but they will also significantly boost the discovery rate of known and unknown rare classes of transient sources throughout the Universe. Among the numerous classes of transients, GRBs have direct links with most of the topics mentioned above. Accurate and systematic GRB polarisation measurements can probe the magnetic field intensity and configuration, which is key to understanding relativistic jet formation and shock acceleration physics. Other unexpected classes that were discovered only a few years ago are FRBs, FBOTs, and other unidentified high-energy transients. The keV-to-MeV energy band is ideally suited to explore high-energy, non-thermal phenomena.

In this White Paper (WP) we discuss how these topics, summarised in the key science questions below, can be addressed by a mission called ASTENA (see WP by [18]). Its payload consists of two instruments: (i) an array of wide-field monitors with imaging, spectroscopic, and polarimetric capabilities (WFM-IS); (ii) a narrow field telescope (NFT) based on a Laue lens operating in the $50-600 \mathrm{keV}$ range with unprecedented angular resolution, polarimetric capabilities, and sensitivity. With respect to THESEUS - aiming at fully exploiting GRBs for early Universe and multi-messenger astrophysics-ASTENA will carry out a deeper study of the physics of their prompt and afterglow emission. Building on the foundation of the THESEUS X-Gamma-ray Imaging Spectrometer (XGIS), ASTENA WFM-IS will have a higher effective area (by a factor of 6), thus providing a substantial step forward for timing, spectroscopy, and polarimetry of GRB prompt emission. The superior 1-arcmin angular resolution of ASTENA WFM-IS is also crucial to perform the NFT follow up of the discovered events.

The key science questions are summarised as follows.

- What is the GRB prompt emission mechanism? What role do magnetic fields play in the jet formation, propagation, energy dissipation, and shock acceleration physics? 
- For electromagnetic counterparts of GW events: what are the jet structure and demographics of short GRBs associated with the merging of BNS?

- What mechanism powers the transient, long-lived hard X/soft $\gamma$-ray emission that characterises GRB afterglows?

- What sources lie behind the number of known transients and unknown X/ $\gamma$-ray transients that have recently been discovered?

\section{GRB prompt emission mechanism and the role of magnetic field}

Technological advancement over the past two decades has revolutionised our understanding of the nature of GRBs (see, e.g., review by [32]), propelling them from mysterious, poorly localised and relatively unstudied, high-energy flashes of unknown origin to the focus of a global scientific community whose efforts have confirmed GRBs to represent the endpoints of stellar evolution and compact binary mergers, drivers of ultrarelativistic plasma outflows and, possibly, key cosmic producers of heavy metals such as gold-all at vast cosmological distance [15] and discovered, localised and followed up in real time.

Despite this rapid progress, the field is in its infancy and fundamental questions on the origin, physics, and impact of GRBs remain. The mechanism responsible for the $\gamma$-ray prompt emission, i.e. the GRB itself, is not yet understood and in many cases cannot be explained with pure optically thin synchrotron. In particular, we do not know how the ultra-relativistic jet is formed near the progenitor, how it propagates through the stellar interior, and the origin and configuration of the associated magnetic field [23], which is a key ingredient for understanding the emission process and the shock acceleration physics [67]. The physical processes, the energy dissipation mechanism, the magnetisation content, and the jet geometry that rule the prompt emission can uniquely be investigated through prompt hard $\mathrm{X} / \gamma$-ray polarisation measurements (e.g., [33]).

Novel instruments on a new generation of fully autonomous, robotic optical telescopes have made rapid early-time optical afterglow polarimetry a reality $[50,51$, 71], revealing a wide range of polarisation properties and confirming the presence of entrained, ordered magnetic fields in some GRB jets. The detection of optical polarisation has regenerated interest in new high-energy polarisation missions (e.g. POLAR, a collaboration between China and Europe, flown on the Chinese Tiangong 2 space lab; XIPE, one of the three candidates for a medium-class mission studied by ESA; and NASA's IXPE mission, scheduled for launch in 2021), despite early controversies and accepted technical challenges in accurately calibrating systematics at $\gamma$-ray energies. The first claim of polarised prompt optical light [75] provides further motivation. To date, no GRB has yet been observed in polarised light from high-energy $\gamma$-rays through X-ray, optical and to radio across prompt, early and late afterglow. Such a dataset would provide unprecedented insight into the physics of GRBs. In addition, although technically more challenging, $\gamma$-ray polarimetry probes directly the prompt emission mechanism and its magnetic field configuration.

According to a recent review [45], previous (CGRO, RHESSI, IKAROS) and current missions (INTEGRAL, Astrosat) reported prompt $\gamma$-ray polarisation 
measurements for ten GRBs. However, the results are highly uncertain for some events, and others have a limited statistical significance $(<4 \sigma)$. Recently, polarisation results have been obtained for 5 bright GRBs (161218A, 170101A, 170127C, 170206A, 170114A) with the POLAR experiment [80]. The systematic errors are certainly better controlled, but the statistical significance, also due to the low polarisation found, is still at about the $3 \sigma$ level. Indeed, the authors state that their analysis does not allow to fully reject the hypothesis that the analysed GRBs are not polarised, even though the observed changes in the polarisation angle suggest a strong temporal evolution as a possible explanation for the low time-averaged polarisation degree. On the basis of the available data, it is difficult to draw definitive conclusions about the underlying physics and emission mechanisms, like the presence of a globally ordered magnetic field in the emission region (e.g., [39]) or the presence of a randomly structured field (e.g., [34]). From the current polarisation measurements it is still not possible to discriminate between emission mechanisms, e.g., between synchrotron in a uniform B-field or in a random B-field, or, for example, the possible presence of a Compton drag. As for the jet structure and geometry, depending on the emission mechanism and on the origin and configuration of the magnetic field, the polarisation level may depend on the viewing angle, i.e. the angle between the line of sight and the jet axis [34]. To draw conclusions about these open issues, one should measure the hard $\mathrm{X} / \gamma$-ray polarisation of a significant sample and correlate with other key properties. For example, [74] derived how the distribution of the polarisation level depends on the intrinsic peak energy $\mathrm{E}_{p}$ of the $E F(E)$ spectrum for three different models (see Fig. 1). To further test some models, it is equally

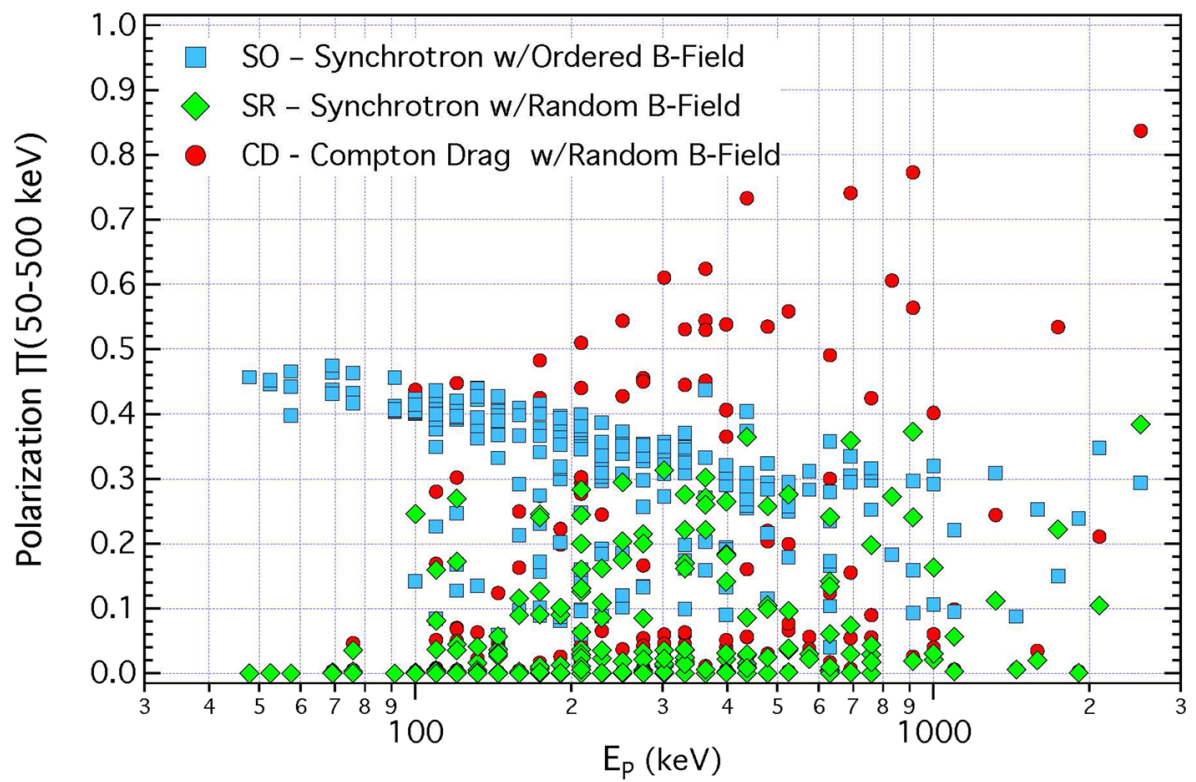

Fig. 1 Distribution of the predicted polarisation level as a function of the intrinsic peak energy $E_{p}$ for three different emission models. The distribution is that reported by [45], that was derived by means of a Montecarlo simulation of 10,000 GRB jets with parameter distributions described by [74] 
important to carry out time-resolved polarisation measurements: for instance, the internal-collision-induced magnetic reconnection and turbulence (ICMART) model predicts decreasing $\gamma$-ray polarisation degree and $E_{\mathrm{p}}$ with time throughout each individual broad $\gamma$-ray pulse [79].

In addition, time-resolved polarisation measurements of GRB prompt emission make it possible to test the weak equivalence principle (WEP) with unprecedented sensitivity: should the WEP be violated, the temporal evolution of the polarisation angle would depend on both photon energy and GRB distance [76].

The ASTENA WFM-IS (see WP by [18]), thanks to its specific capability as a polarimeter, its very large detection area and broad passband, is expected to provide accurate information about the polarisation level of a large sample of GRBs, also determining its temporal and energy dependence. We estimated the minimum detectable polarisation (MDP) as a function of photon energy, for a typical long GRB with a fluence of $\sim 10^{-4} \mathrm{erg} \mathrm{cm}^{-2}$ : as shown in Fig. 2, the MDP is $<10 \%$ for $E<300 \mathrm{keV}$, and $<30 \%$ for $E<600 \mathrm{keV}$. The broad passband is key to cover $E_{\mathrm{p}}$ for most GRBs, not only because most of the energy is emitted around $E_{\mathrm{p}}$, but also because it makes it possible to discriminate between competing models based on the polarisation degree vs. $E_{\mathrm{p}}$ distribution, and to test predictions on spectro-polarimetric evolution patterns. By way of example, with reference to Fig. 1, detecting 50\% polarisation around $400 \mathrm{keV}$ would rule out both synchrotron models considered.

\section{Electromagnetic counterparts of gravitational wave events}

The observation of the gravitational wave event GW 170817 by the LIGO-Virgo Collaboration (LVC) and the discovery of the short GRB 170817A $\sim 1.7$ s later and

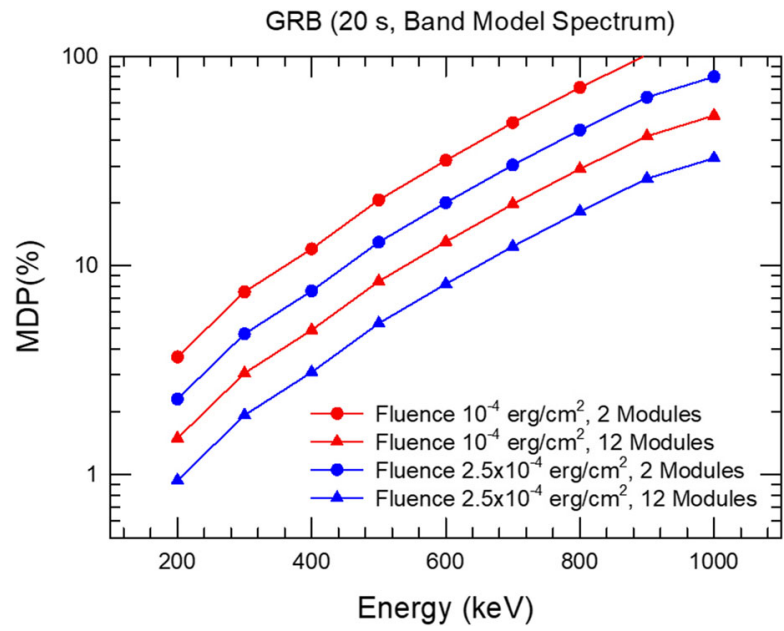

Fig. 2 Minimum detectable polarisation with ASTENA WFM-IS for a 20-s long GRB, with different fluence values, and for two different number of modules. The GRB spectrum is described by the Band function with typical values $\left(\alpha=-1, \beta=-2.3, E_{\mathrm{p}}=300 \mathrm{keV}\right)$. Reprinted from [18] 
positionally consistent with the location uncertainty $\left(31 \mathrm{deg}^{2}\right)$ of the GW event has ushered in the multi-messenger astronomy era [1]. Finding the EM counterpart of a GW event is crucial (1) to provide independent confirmation of the astrophysical origin of the GW trigger, especially for GW events with low significance (e.g., subthreshold triggers), (2) to accurately determine the position in the sky, and therefore (3) to allow for the host galaxy identification and redshift determination. As a consequence, this information can break the degeneracy between position in the sky and other observables, both extrinsic (binary inclination and GW polarisation angles, distance) and intrinsic (orbital angular momentum, spin, see [55]). In addition, it makes it possible to independently estimate the Hubble constant (e.g., [25]) and to investigate fundamental physics, like the Lorentz Invariance violation foreseen in quantum gravity theories (e.g., [4]).

On the basis of the results obtained from the detection of the short GRB 170817A and the observing campaign that led to the discovery of the kilonova $(\mathrm{KN})$ associated with GW 170817 in the outskirts of the galaxy NGC 4993 at a distance of $40 \mathrm{Mpc}$ from the Earth, a few important properties are worth mentioning: a) GRB 170817A is sub-luminous $\left(\sim 1 \times 10^{47} \mathrm{erg} \mathrm{s}^{-1}\right)$ [22]; b) it is softer than typical short GRBs (sGRBs), as demonstrated by its non-detection (upper limit of $10^{-7} \mathrm{erg} \mathrm{cm}^{-2} \mathrm{~s}^{-1}$ in $0.2-5 \mathrm{MeV}$ ) with the very large effective area High Energy (HE) instrument aboard the Insight-HXMT mission [35]; c) a very rough localisation accuracy of the event ( $90 \%$ probability region of about 1800 square degrees) obtained with the GBM instrument aboard the Fermi satellite. This very rough localisation accuracy, combined with the low hard X-ray flux, prevented an accurate spectral analysis and an immediate multiwavelength follow-up of GRB 170817A for the study of the afterglow emission.

The broadband study of the afterglow of GRB 170817A allowed us to model the jet geometry, the kinetic energy and bulk velocity angular structure, raising the following questions: is the jet structure universal? Which parameters are involved? To answer these, it is worth noting that the presence of a universally structured jet, whose origin is directly related to the central engine and its interaction with the ambient medium (e.g. [64]), could also be assessed through prompt emission observations and population studies $[56,63]$.

All the above sGRB properties and instrument limitations could be ideally addressed with an instrument with a much more sensitive wide-FOV monitor, a very broad energy passband that extends to low $\mathrm{X}$-ray energies $(\sim 1 \mathrm{keV})$, and with spectroscopic and possibly polarimetric capabilities. The WFM-IS proposed by us matches all these requirements. Compared with THESEUS-XGIS, which operates in the same broad energy band, the WFM-IS has a larger FOV ( 2 sr vs. 1.5 sr), significantly larger effective area (by a factor of $\sim 6$ ) and, most importantly, better point source localisation accuracy ( 1 vs. several arcmin), which is crucial to allow for a rapid followup of focusing telescopes, such as the NFT.

In the 2030's, Third Generation (3G) GW detectors such as the Einstein Telescope (ET) and the Cosmic Explorer (CE) will be operational. The ET will detect $>10^{4} \mathrm{yr}^{-1}$ BNS mergers out to $z \sim 2$ [59], of which a few tens will also be detected with THESEUS-XGIS as sGRBs. On the optical side, the Rubin Observatory will not detect kilonova emission beyond $z \sim 0.5$. Therefore, the combination of larger 
effective area and broad band of WFM-IS is essential to fully exploit the potential of $\mathrm{ET}+\mathrm{CE}$ for prompt localisation and characterisation of the associated EM emission for more BNS mergers. This, in turn, will boost the population study. The distribution of observed GW-EM delays, combined with the spectral characterisation and $\gamma$-ray luminosity of the associated sGRB, will further constrain the jet geometry, structure, and finally the formation channel(s) of these compact binary systems.

The mentioned GRB properties are those found in the case of a GW event due to a BNS merger. NS-BH are expected to produce different EM counterparts (GRB, kilonova, afterglow-e.g., [6]). However, their luminosity and evolutionary timescales are still observationally unexplored.

Lastly, the Laser Interferometer Space Antenna (LISA), that will be observing GWs in the millihertz band, will detect thousands of stellar-mass binary black holes (BBH) with $M>30 M_{\odot}$ at $z<0.5$, hundreds of which will be discovered years before coalescence. It will predict the time of merging with $<10 \mathrm{~s}$ uncertainty and $<1 \mathrm{deg}^{2}$ position error [46, 66]. A sensitive, broadband, $\mathrm{X} / \gamma$-ray monitor with a sufficient FOV such as the ASTENA WFM-IS is key to monitor a possible prompt EM counterpart.

\section{Hard X-ray spectrum and polarisation of GRB afterglows}

Thanks to the BeppoSAX mission that discovered GRB afterglows and the Neil Gehrels Swift observatory that initiated the systematic study of the early afterglow, we have a very consolidated knowledge of the temporal and spectral behaviour of the low energy $(<10 \mathrm{keV})$ afterglow (see, e.g., [32, 77]). However, due to the limited sensitivity of the current instrumentation, at higher energies the afterglow temporal and spectral behaviour is an almost uncharted territory. High energy X-ray measurements have been obtained only by the BeppoSAX Phoswich Detection System (PDS) [40], NUSTAR [30], or INTEGRAL [44]. The PDS telescope detected the high energy afterglow of GRB 990123, one of the brightest GRBs observed with BeppoSAX. The afterglow was detected up to $60 \mathrm{keV}$, with a power-law decay in the $15-28 \mathrm{keV}$ that was consistent with that found in the $2-10 \mathrm{keV}$ band (slope $\alpha=1.46 \pm 0.04$ ) at least up to $12 \mathrm{~h}$ after the event. Concerning the afterglow spectrum between 6 and $11 \mathrm{~h}$ from the GRB onset, it was found that from 0.6 to $60 \mathrm{keV}$ it was well fit with an absorbed power-law with the multiwavelength spectral energy distribution (SED) shown in Fig. 3. [14] discussed the BeppoSAX results finding that, while the temporal and spectral behaviour of the optical afterglow is consistent with synchrotron with cooling frequency between the optical and the X-rays as long as the $2-10 \mathrm{keV}$ data are considered, this is no longer the case when the spectrum is extended up to $60 \mathrm{keV}$. In this case an Inverse Compton (IC) scattering was proposed to explain the BeppoSAX results. The importance of the hard $\mathrm{X}$-ray detection for establishing the afterglow emission mechanism at play is confirmed by the results obtained by [30], who detected the hard X-ray afterglow from GRB 130427A with NuSTAR. The result was that "the NUSTAR data are essential in constraining the shape of the broadband spectra". In this case the NUSTAR power-law spectrum and decay law are still 

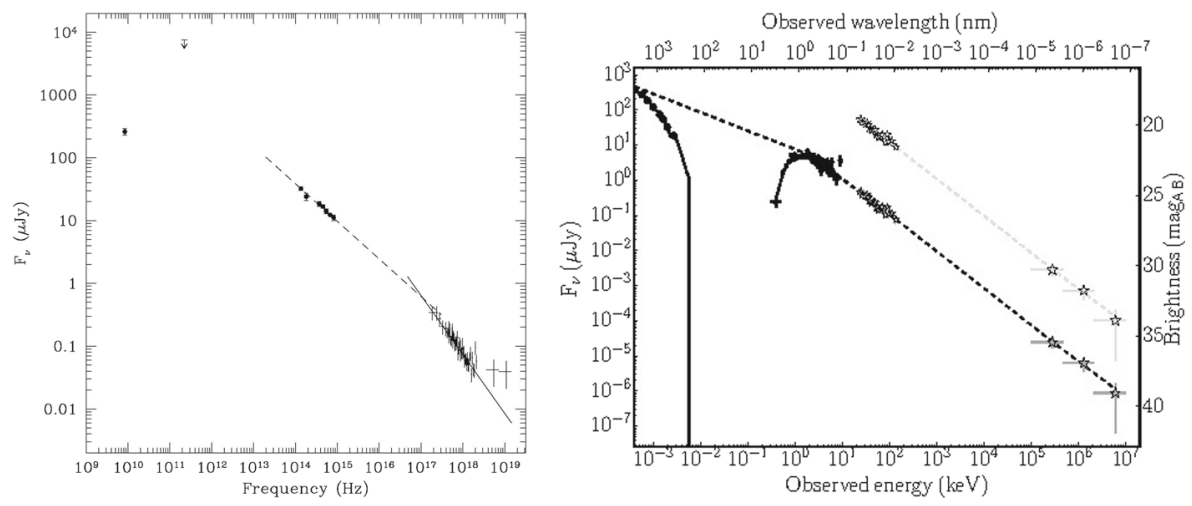

Fig. 3 Left: spectral Energy Distribution (SED) of the afterglow emission of GRB 990123. Reprinted from [40]. Right: same for the afterglow of GRB 120711. The same spectral regime spans the X-ray-to-GeV energy range (reprinted from [44])

consistent with a synchrotron emission mechanism of the GRB afterglow. Analogous results and conclusions were also obtained for the bright GRB 120711A [44].

For a few GRBs, whose $0.3-10 \mathrm{keV}$ afterglow was found to be exceptionally soft (power-law photon index $\Gamma_{X}>3$ ), [48] found that an additional, hard X-ray component of unknown origin and modelled as a hard power-law, was required by the data. Such a component could be more ubiquitous than what has been observed with Swift-XRT; only when the dominant component is particularly soft, as in the case of these GRBs, would the hard one become detectable.

In the cases of some late X-ray afterglows, the combination of (i) slow decay, (ii) very soft photon index $\left(\Gamma_{X}>3\right.$, inconsistent with a pure synchrotron origin), (iii) larger-than-average intrinsic absorption, and (iv) very long $\left(T_{90}>1000 \mathrm{~s}\right)$ prompt $\gamma$-ray emission, was observed [42]. The negligible chance probability of a random combination of all these rare properties suggested that the extremely soft X-ray emission could be due to reprocessing by the complex circumburst environment sculpted by the progenitor prior to the final explosion. A hard $\mathrm{X} / \gamma$-ray characterisation of the afterglow is missing and it would help test this interpretation.

Fermi/LAT unveiled the presence of the afterglow emission at very high energies [7, 20]. So far it detected long-lived emission in the $100 \mathrm{MeV}-100 \mathrm{GeV}$ range for almost 200 GRBs [3]. Combining Swift/XRT and LAT data, [2] found that the presence of a synchrotron cooling break between $\mathrm{X}$-rays and $\mathrm{GeV}$ can explain the non-detection by LAT of relatively bright X-ray afterglows, whereas LAT-detected GRBs are mostly consistent with a synchrotron spectrum whose cooling break lies either below X-rays or above the LAT passband (see Fig. 4). This, in turn, suggests that LAT-detected GRBs occur in wind-profile, low-density circumburst environments. No evidence is found for a dominant synchrotron self-Compton (SSC) component in the LAT range (with implications on the relative strength of the shock microphysics parameters describing the magnetic field vs. electron energy), where the possibility remains that such an SSC component is detectable below $100 \mathrm{MeV}$. 


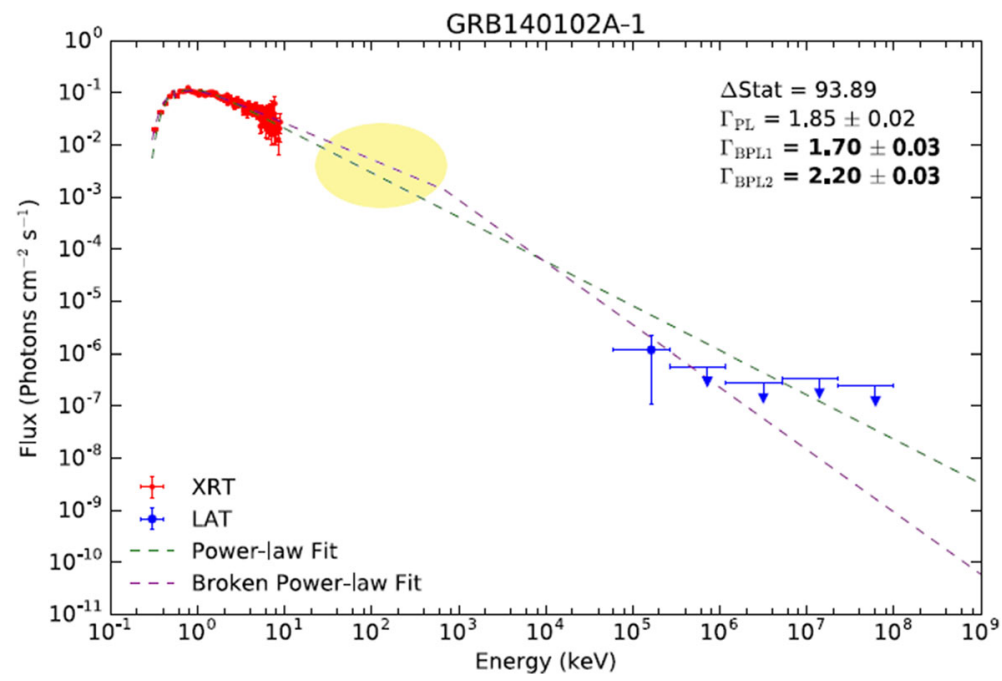

Fig. 4 Example of an X-ray-to- $\gamma$-ray SED of the afterglow of GRB 140102A based on Swift/XRT and Fermi/LAT data. The yellow area highlights where ASTENA/NFT observations are key to constrain the presence of synchrotron cooling break (adapted from [2])

These few observations clearly show the importance of filling the energetic gap between X-rays and the LAT passband in the afterglow study: this would help shed light on the nature of the very high-energy early afterglow and on the possible role of pairs $[9,20]$. ASTENA, thanks to the unprecedented sensitivity of the Narrow Field Telescope (NFT) (see [18]) will be capable to measure the high energy spectrum and the polarisation [11] of the afterglow emission of a large sample of GRBs, and thus to establish the underlying physics and the emission mechanisms in play (e.g., synchrotron photon energy, presence of a Compton or pair production component in addition to the synchrotron) and the corresponding parameters.

\section{Low luminosity GRBs}

Low luminosity GRBs (llGRBs) are a sub-population of long GRBs. They are single peaked, smooth and in most cases soft, with luminosity in the range $10^{47}-10^{49} \mathrm{erg}$, much lower than typical GRBs. Most of them (about a dozen) are associated with type Ic supernovae (SNe). The prototype of the $l l \mathrm{GRBs}$ is GRB 060218, from which evidence of a relativistic shock-breakout (SBO) of the stellar envelope and the stellar wind was observed [10]. However, a few of them do not have an associated SN, e.g., GRB 060505 [19]. These properties place $l l \mathrm{GRBs}$ between the highly relativistic, collimated GRB explosions and the spherical, ordinary Type Ib/c SNe as it is shown in Fig. 5 (reprinted from [41]), which compares the kinetic energy distribution (or profile) versus the outflow velocity for different classes of sources. As suggested by this figure and as it was discussed by [41], the kinetic energy profile of $l l \mathrm{GRBs}$ 


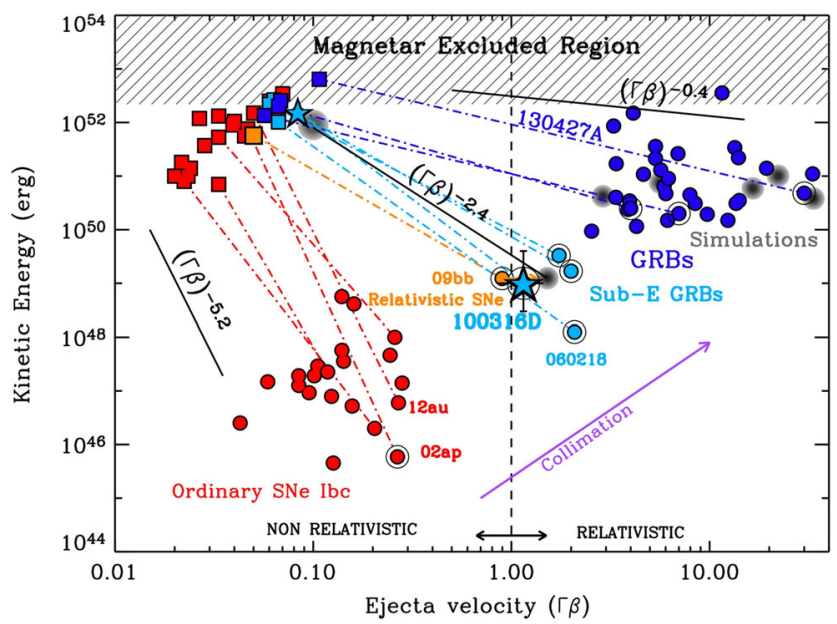

Fig. 5 Kinetic energy behaviour of different classes of transient sources, i.e. ordinary SNe type Ibc, llGRBs (here shown as "Sub-E GRBs") and classical GRBs (reprinted from [41])

is intermediate between that of ordinary, non-relativistic SNe Ibc and that of classical GRBs. This behaviour of $l l$ GRBs calls for the presence of a central engine that drives a jet which either gets choked or is barely able to reach the star surface. In the relativistic SBO interpretation [54], three observables (duration, SBO energy, and SBO temperature) can be used to measure the SBO radius, thus making inferences on the progenitor's nature. In addition, the so-called relativistic SBO relation must be satisfied by the same observables:

$$
\frac{t_{\mathrm{bo}}^{\mathrm{obs}}}{20 \mathrm{~s}} \sim\left(\frac{E_{\mathrm{bo}}}{10^{46 \mathrm{erg}}}\right)^{1 / 2}\left(\frac{T_{\mathrm{bo}}}{50 \mathrm{keV}}\right)^{-2.68}
$$

This way, one can explain both typical long-soft events and less common, relatively short ( $\sim 30 \mathrm{~s})$ and hard $\left(E_{\mathrm{p}} \sim 50 \mathrm{keV}\right)$ ones, such as GRB 980425 [31, 58]. Alternatively, $l l \mathrm{GRB}$ s could be the natural extension of cosmological GRBs in a universal structured jet scenario [56, 63].

All observed $l l$ GRBs are at low redshifts $(\leq 1)$. Due to their low luminosities, with the current instrumentation they could not have been detected from further out. However, taking into account the relatively small sampled volume, their inferred volumetric rate could be much larger than that of typical long GRBs [24, 36, 69]. So a much more sensitive wide field monitor than the current GRB instrumentation is needed to perform a sensitive sky survey of $l l \mathrm{GRBs}$.

\section{Fast blue optical transients}

Fast blue optical transients (FBOTs) are likely a new class of transient sources [17], which typically show blue spectra, can be as luminous as $\mathrm{SNe}-\mathrm{Ibc}$ ( $\mathrm{L}_{\text {peak }}>$ 
$10^{43} \mathrm{erg} / \mathrm{s}$ ), but evolve more rapidly ( $t_{\text {rise }} \ll 4$ days), in tension with the powering of ${ }^{56} \mathrm{Ni}$ decay as prescribed by traditional SN models $[60,62]$. At the moment only a very few objects of this class have been detected in X-rays, and AT2018cow, discovered in the optical band [68], is by far the best studied yet, also thanks to its vicinity $(60 \mathrm{Mpc})$. AT2018cow was found to fade very rapidly [43], much more than all types of SNe (see left panel of Fig. 6) with a peak luminosity of about $4 \times 10^{44} \mathrm{erg} / \mathrm{s}$. Yet, from the star formation properties of the host dwarf galaxy and the site of AT2018cow within the host, a massive star origin is favoured [49]. Its X-ray counterpart, detected with the Swift XRT, NUSTAR and INTEGRAL [43], faded in the 0.5-10 keV band faster than typical GRB afterglows, more similar to that of tidal disruption events (TDEs; see right panel of Fig. 6). The very interesting peculiarity of AT2018cow in the X-ray band is its low-energy power-law spectrum that extends to hard X-rays with a very broad bump that fades away at $\sim 20$ days. This feature is uncommon in transient objects.

AT2018cow is likely the prototype of a new class of transients that are expected to be copiously detected in the optical band thanks to the current and forthcoming wide-field surveys. Yet, the following questions remain unanswered: which is the progenitor of this class of transients? Are they related to GRBs or TDEs? In this case they should show a prompt emission, which is as yet undetected. How common is the hard X-ray component and what is its origin?

Margutti et al. [43] propose a Compton-disc reflection model as the origin of the AT2018cow hard X-ray bump, at 7.7 days from the optical discovery. Only numerous detections in the future and more sensitive hard X-ray observations can clarify the origin of the FBOT emission and their progenitors.
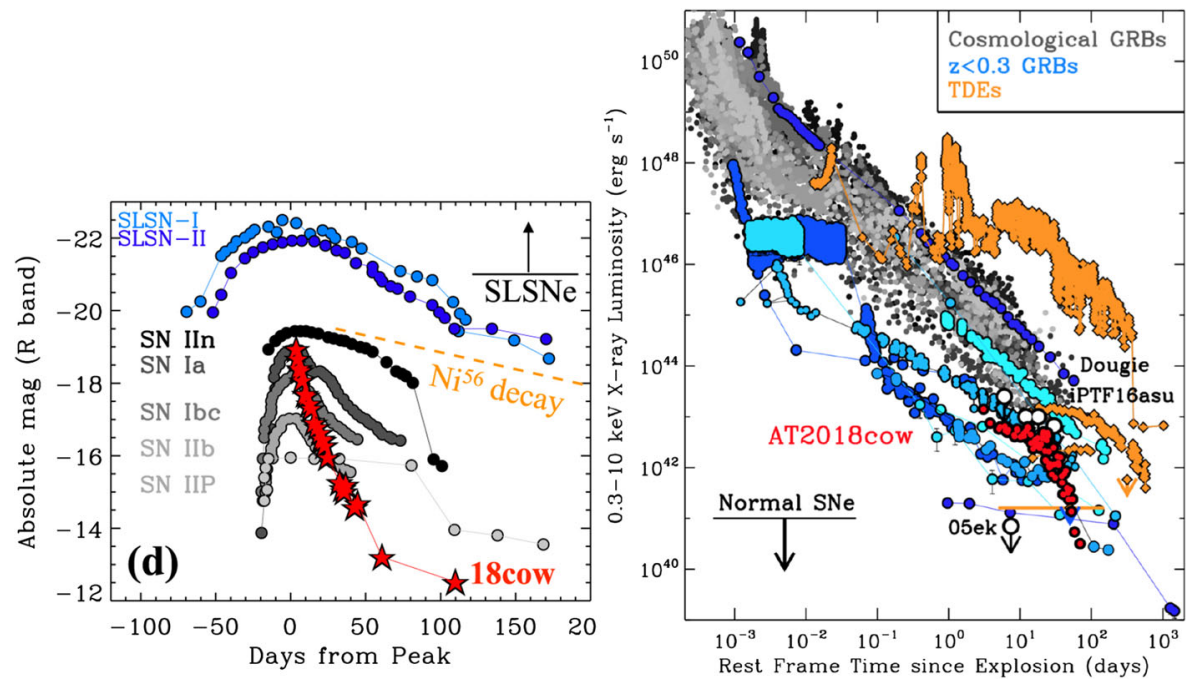

Fig. 6 Left: Comparison of the optical light curve of AT2018cow with those of the various types of SNs. Right: Comparison of the X-ray fading of AT2018cow with those of GRBs and TDEs. Reprinted from [43] 


\section{Fast radio bursts}

In addition to the possible class of the $\mathrm{X} / \gamma$-ray counterparts of the FBOTs, another class of transient events could exhibit an associated $\mathrm{X} / \gamma$-ray emission: fast radio bursts (FRBs). Discovered in 2007 [37], FRBs are still one of the most intriguing mysteries in astrophysics (see [29, 57] for recent reviews). They are very short ( $1 \mathrm{~ms}$ ) and are distributed randomly over the sky. Although the number of FRBs publicly announced so far is still limited ( $~ 80$ as of July 2019$)$, there is no concentration of FRB in the Galactic plane, and thus they are very likely isotropically distributed over the sky. Their extragalactic origin is based on the detection of a repeating FRB (121102, [70]), that has allowed to accurately determine the FRB direction and thus its association with a persistent radio source [12], coincident with a bright star-forming region located in the outskirts of an irregular, low-metallicity dwarf galaxy with a redshift $z=0.193$ [73]. Very recently, the redshifts of two other non-repeating FRBs were obtained: FRB 180924 at $z=0.3214$ [5] and FRB 190523 at $z=0.66$ [61]. Meanwhile, another repeating FRB (180814) has been detected [13]. Their dispersion measures, if assumed to be produced mostly in the intergalactic medium, can be used as a proxy of their distances: under this assumption, their luminosities are in the range $10^{38}-10^{43} \mathrm{erg} / \mathrm{s}$, which are much lower than those found for GRBs, even $l l G R B s$. This fact would mean that FRBs are much less energetic than other astrophysical transients, and thus their counterparts are hardly detectable, unless they are many orders of magnitude more energetic than the FRBs themselves. Actually, controversial evidence was found of a putative Swift GRB spatially and temporally coincident with FRB 131104 [16], although no simultaneous X-/ $\gamma$-ray counterparts to other much more radio bright FRBs were found [26, 65, 72]. Several models that have been proposed in the literature, (e.g., [47, 52, 53, 78] and references therein) do not exclude the possibility of $\gamma$-ray emission associated with FRBs. Therefore, it is very important to have a very sensitive instrument with imaging capabilities that can detect possible $\gamma$-ray counterparts of FRBs.

\section{Other unknown high-energy transients}

A number of extragalactic $\mathrm{X}$-ray transients has been discovered in recent years, with luminosities in the range $10^{40}-10^{46} \mathrm{erg} / \mathrm{s}$ and duration of several seconds to hours. Their origin remains mysterious and while several interpretations remain plausible, none of them appears to be convincing. Hereafter, we briefly review some of them as a few examples.

An X-ray flash was observed with $L=6 \times 10^{42} \mathrm{erg} / \mathrm{s}$. It lasted $4000 \mathrm{~s}$ and was found in the elliptical galaxy M86 without any apparent hard X/ $\gamma$-ray emission ([28]; see also [21, 38]). It could be the result of a tidal disruption of a white dwarf by an intermediate $\mathrm{BH}$ or an off-axis short GRB.

Ultraluminous X-ray flares with luminosities in the range $10^{39}-10^{41} \mathrm{erg} / \mathrm{s}$ were observed repeatedly from extragalactic X-ray sources, that otherwise look like compact X-ray binaries [27]. They showed a fast rise (about one minute), followed by 
$\sim 10^{3}$ s decay. Although the flaring and energetic profile are reminiscent of Galactic magnetars, they are located in old stellar population regions.

Another X-ray transient was discovered serendipitously in the Chandra Deep Field-South [8], showing a comparable rise time, followed by a power-law decay. Its energy spectrum is modelled with a relatively hard power-law, with photon index $\Gamma_{X}=1.43_{-0.13}^{+0.23}$. The peak luminosity lies in the range $10^{44}-10^{47} \mathrm{erg} / \mathrm{s}$, depending on the unknown distance. While most of the previously known X-ray transients are ruled out, the following possible interpretations remain plausible: $\mathrm{X}$-ray afterglow of either (i) an off-axis sGRB or (ii) a $l l$ GRB at $z \gtrsim 2$ with no prompt emission below $20 \mathrm{keV}$; (iii) a highly beamed TDE of a white dwarf disrupted by an intermediate black hole. The estimated rate of these $\mathrm{X}$-ray transients lies in the range from a few up to $10^{4} \mathrm{Gpc}^{-3} \mathrm{yr}^{-1}$ [8].

These examples of unknown X-ray transients, whose rates in the local Universe could be comparable with that of core-collapse SNe, show how a broadband, more sensitive, survey instrument such as the ASTENA WFM-IS would significantly contribute to boost their discovery rate and and to characterise them. In addition, follow-up observations with the ASTENA NFT would help further characterise their long-lasting emission and decay.

\section{ASTENA mission concept}

For a detailed description of the ASTENA scientific payload, capabilities and expected performance, the reader is referred to the companion WP by [18] devoted to the science cases that can be addressed with ASTENA in the nuclear astrophysics domain.

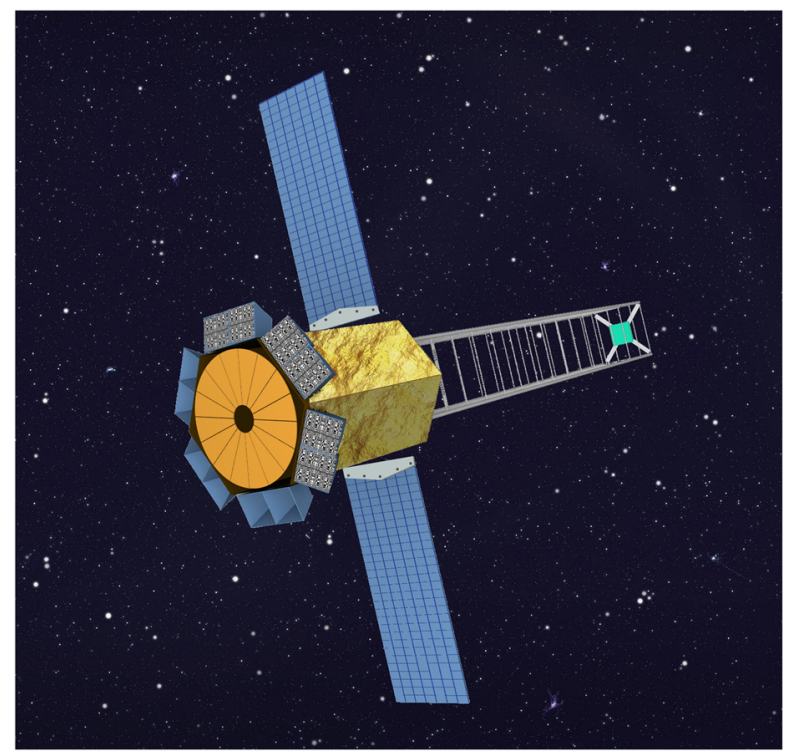

Fig. 7 Artistic view of ASTENA in-flight configuration 
Table 1 Main properties of the ASTENA scientific payload

\begin{tabular}{|c|c|c|}
\hline & WFM-IS & NFT \\
\hline Energy pass-band & $2 \mathrm{keV}-20 \mathrm{MeV}$ & $50-600 \mathrm{keV}$ \\
\hline \multirow[t]{3}{*}{ Total useful area ${ }^{\mathrm{a}}$} & $\sim 5800 \mathrm{~cm}^{2}(<30 \mathrm{keV})$ & \\
\hline & $\sim 6700 \mathrm{~cm}^{2}(30-150 \mathrm{keV})$ & $7 \mathrm{~m}^{2}$ (projected) \\
\hline & $\sim 13800 \mathrm{~cm}^{2}(>200 \mathrm{keV})$ & \\
\hline Field of View & $2 \mathrm{sr}$ & $4 \operatorname{arcmin}$ \\
\hline Angular resolution & $6 \operatorname{arcmin}$ & $\sim 30 \operatorname{arcsec} \mathrm{HPD}$ \\
\hline Point source localization accuracy & $1 \operatorname{arcmin}$ & $<10 \operatorname{arcsec}$ \\
\hline Continuum sensitivity & see Figure 11 of [18] & see Figure 15 of [18] \\
\hline Minimum Detectable Polarization & see Fig. 2 & see Figure 16 of [18] \\
\hline
\end{tabular}

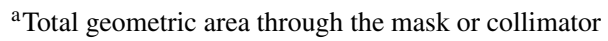

Briefly, the ASTENA in-flight configuration is shown in Fig. 7. The instrumentation on board consists of a Wide Field Monitor-Imaging Spectrometer (WFM-IS) with a $2 \mathrm{keV}-20 \mathrm{MeV}$ passband, and a Narrow Field Telescope (NFT) with a 50-600 keV passband. The WFM-IS consists of an array of 12 units, two units per each side of the hexagon, that surround the NFT. All the units are offset by $15 \mathrm{deg}$ with respect to the axis of the NFT, as shown in Fig. 7. The NFT is a Laue lens telescope of about $3 \mathrm{~m}$ diameter and $20 \mathrm{~m}$ focal length. The latter is partly (5 out of $20 \mathrm{~m}$ ) inside the spacecraft. Table 1 summarises the main properties of the ASTENA payload.

\section{Conclusions}

The coming decades promise truly comprehensive multi-messenger exploration of the transient sky due to (1) transformational ground- and space-based technologies that will probe the gravitational-wave Universe across a broad frequency range at unprecedented sensitivities, (2) a step-change in sensitivity and capability from new ground-based astro-particle facilities, and (3) a revolution in real-time discoveryat scale-with complementary wide-field, high-cadence and sensitive surveys across the entire electromagnetic spectrum. The data rates will transform how astronomy is conducted and open a new era of 'big' data science and a corresponding innovation revolution in artificial intelligence and machine learning.

The ESA Science Programme "Voyage 2050" offers the opportunity to explore uncharted territories as well as tackle open issues in astrophysics, cosmology, and fundamental physics from the high-energy transient sky perspective, during this unique and imminent period of technological development and wide-reaching synergy.

In this article, we focused on major, fundamental but unsolved issues in the field of the high-energy transient sky, which can be addressed with the proposed ASTENA mission: 1) the origin of GRB prompt emission and the role of magnetic fields in the jet formation and energy dissipation; 2) the jet structure and demographics of short 
GRBs associated with the merging of binary neutron star mergers; 3) the nature of the radiation process that powers GRB high-energy afterglows; 4) the identification of the plethora of known and unknown $\mathrm{X} / \gamma$-ray transient sources that have recently been discovered and those that will be discovered copiously in the future.

In short, ASTENA will be a breakthrough facility for high-energy astrophysics. Its broad band ( $2 \mathrm{keV}-20 \mathrm{MeV}$ ) coverage and payload of two instruments- the WFM-IS and its focusing NFT with a 50-600 keV passband-will together provide unprecedented sensitivity to continuum and line emission, unique new polarimetric capabilities, and outstandingly high $\lesssim 1^{\prime}$ angular resolution, all in one mission. Furthermore, it will drive breakthroughs in the nuclear astrophysics field and will establish the origin of the 511-keV positron annihilation line observed from the Galactic Centre, as described in the companion White Paper by [18].

Acknowledgements The ASTENA mission concept is the result of several development activities. We wish to acknowledge the support by the Italian Space Agency (ASI) and that by the AHEAD European program.

Funding Open access funding provided by Università degli Studi di Ferrara within the CRUI-CARE Agreement.

Open Access This article is licensed under a Creative Commons Attribution 4.0 International License, which permits use, sharing, adaptation, distribution and reproduction in any medium or format, as long as you give appropriate credit to the original author(s) and the source, provide a link to the Creative Commons licence, and indicate if changes were made. The images or other third party material in this article are included in the article's Creative Commons licence, unless indicated otherwise in a credit line to the material. If material is not included in the article's Creative Commons licence and your intended use is not permitted by statutory regulation or exceeds the permitted use, you will need to obtain permission directly from the copyright holder. To view a copy of this licence, visit http://creativecommons.org/licenses/by/4.0/.

\section{References}

1. Abbott, B.P., Abbott, R., Abbott, T.D., Acernese, F., Ackley, K., Adams, C., Adams, T., Addesso, P., Adhikari, R.X., Adya, V.B., et al.: Multi-messenger observations of a binary neutron Star Merger. ApJ 848, L12 (2017). https://doi.org/10.3847/2041-8213/aa91c9

2. Ajello, M., Baldini, L., Barbiellini, G., Bastieri, D., Bellazzini, R., Bissaldi, E., Blandford, R.D., Bonino, R., Bottacini, E., Bregeon, J.: Investigating the nature of late-time high-energy GRB emission through joint Fermi/Swift observations. ApJ 863(2), 138 (2018). https://doi.org/10.3847/1538-4357/ aad000

3. Ajello, M., Arimoto, M., Axelsson, M., Baldini, L., Barbiellini, G., Bastieri, D., Bellazzini, R., Bhat, P.N., Bissaldi, E., Blandford, R.D., Bonino, R., Bonnell, J., Bottacini, E., Bregeon, J., Bruel, P., Buehler, R., Cameron, R.A., Caputo, R., Caraveo, P.A., Cavazzuti, E., Chen, S., Cheung, C.C., Chiaro, G., Ciprini, S., Costantin, D., Crnogorcevic, M., Cutini, S., Dainotti, M., D’Ammando, F., de la Torre Luque, P., de Palma, F., Desai, A., Desiante, R., Di Lalla, N., Di Venere, L., Fana Dirirsa, F., Fegan, S.J., Franckowiak, A., Fukazawa, Y., Funk, S., Fusco, P., Gargano, F., Gasparrini, D., Giglietto, N., Giordano, F., Giroletti, M., Green, D., Grenier, I.A., Grove, J.E., Guiriec, S., Hays, E., Hewitt, J.W., Horan, D., Jóhannesson, G., Kocevski, D., Kuss, M., Latronico, L., Li, J., Longo, F., Loparco, F., Lovellette, M.N., Lubrano, P., Maldera, S., Manfreda, A., Martí-Devesa, G., Mazziotta, M.N., Mereu, I., Meyer, M., Michelson, P.F., Mirabal, N., Mitthumsiri, W., Mizuno, T., Monzani, M.E., Moretti, E., Morselli, A., Moskalenko, I.V., Negro, M., Nuss, E., Ohno, M., Omodei, N., Orienti, M., Orlando, E., Palatiello, M., Paliya, V.S., Paneque, D., Persic, M., Pesce-Rollins, M., Petrosian, V., Piron, F., Poolakkil, S., Poon, H., Porter, T.A., Principe, G., Racusin, J.L., Rainò, S., Rando, R., Razzano, M., 
Razzaque, S., Reimer, A., Reimer, O., Reposeur, T., Ryde, F., Serini, D., Sgrò, C., Siskind, E.J., Sonbas, E., Spandre, G., Spinelli, P., Suson, D.J., Tajima, H., Takahashi, M., Tak, D., Thayer, J.B., Torres, D.F., Troja, E., Valverde, J., Veres, P., Vianello, G., von Kienlin, A., Wood, K., Yassine, M., Zhu, S., Zimmer, S.: A decade of gamma-ray bursts observed by Fermi-LAT: the second GRB catalog. ApJ 878(1), 52 (2019). https://doi.org/10.3847/1538-4357/ab1d4e

4. Amelino-Camelia, G., Ellis, J., Mavromatos, N.E., Nanopoulos, D.V., Sarkar, S.: Tests of quantum gravity from observations of $\gamma$-ray bursts. Nature 395, 525 (1998). https://doi.org/10.1038/26793

5. Bannister, K.W., Deller, A.T., Phillips, C., Macquart, J.P., Prochaska, J.X., Tejos, N., Ryder, S.D., Sadler, E.M., Shannon, R.M., Simha, S., Day, C.K., McQuinn, M., North-Hickey, F.O., Bhandari, S., Arcus, W.R., Bennert, V.N., Burchett, J., Bouwhuis, M., Dodson, R., Ekers, R.D., Farah, W., Flynn, C., James, C.W., Kerr, M., Lenc, E., Mahony, E.K., O’Meara, J., Osłowski, S., Qiu, H., Treu, T., U, V., Bateman, T.J., Bock, D.C.J., Bolton, R.J., Brown, A., Bunton, J.D., Chippendale, A.P., Cooray, F.R., Cornwell, T., Gupta, N., Hayman, D.B., Kesteven, M., Koribalski, B.S., MacLeod, A., McClure-Griffiths, N.M., Neuhold, S., Norris, R.P., Pilawa, M.A., Qiao, R.Y., Reynolds, J., Roxby, D.N., Shimwell, T.W., Voronkov, M.A., Wilson, C.D.: A single fast radio burst localized to a massive galaxy at cosmological distance. Science 365(6453), 565-570 (2019). https://doi.org/10.1126/science.aaw5903

6. Barbieri, C., Salafia, O.S., Perego, A., Colpi, M., Ghirlanda, G.: Light-curve models of black holeneutron star mergers: steps towards a multi-messenger parameter estimation. A\&A 625, A152 (2019). https://doi.org/10.1051/0004-6361/201935443

7. Barniol Duran, R., Kumar, P.: Implications of electron acceleration for high-energy radiation from gamma-ray bursts. MNRAS 412(1), 522-528 (2011). https://doi.org/10.1111/j.1365-2966.2010. 17927.x

8. Bauer, F.E., Treister, E., Schawinski, K., Schulze, S., Luo, B., Alexander, D.M., Brandt, W.N., Comastri, A., Forster, F., Gilli, R., Kann, D.A., Maeda, K., Nomoto, K., Paolillo, M., Ranalli, P., Schneider, D.P., Shemmer, O., Tanaka, M., Tolstov, A., Tominaga, N., Tozzi, P., Vignali, C., Wang, J., Xue, Y., Yang, G.: A new, faint population of X-ray transients. MNRAS 467(4), 4841-4857 (2017). https://doi.org/10.1093/mnras/stx417

9. Beloborodov, A.M., Hascoët, R., Vurm, I.: On the origin of GeV emission in gamma-ray bursts. ApJ 788(1), 36 (2014). https://doi.org/10.1088/0004-637X/788/1/36

10. Campana, S., Mangano, V., Blustin, A.J., Brown, P., Burrows, D.N., Chincarini, G., Cummings, J.R., Cusumano, G., Della Valle, M., et al.: The association of GRB 060218 with a supernova and the evolution of the shock wave. Nature 442, 1008-1010 (2006). https://doi.org/10.1038/nature04892

11. Caroli, E., Curado da Silva, R.M., Stephen, J.B., Pisa, A., Auricchio, N., Del Sordo, S., Donati, A., Schiavone, F., Landini, G., Honkimaki, V., Frontera, F.: A polarimetric experiment with a Laue lens and CZT pixel detector. IEEE Trans. Nuclear Sci. 56(4), 1848-1854 (2009). https://doi.org/10.1109/TNS.2009.2021475

12. Chatterjee, S., Law, C.J., Wharton, R.S., Burke-Spolaor, S., Hessels, J.W.T., Bower, G.C., Cordes, J.M., Tendulkar, S.P., Bassa, C.G., Demorest, P., Butler, B.J., Seymour, A., Scholz, P., Abruzzo, M.W., Bogdanov, S., Kaspi, V.M., Keimpema, A., Lazio, T.J.W., Marcote, B., McLaughlin, M.A., Paragi, Z., Ransom, S.M., Rupen, M., Spitler, L.G., van Langevelde, H.J.: A direct localization of a fast radio burst and its host. Nature 541, 58-61 (2017). https://doi.org/10.1038/nature20797

13. CHIME/FRB Collaboration, Amiri, M., Bandura, K., Bhardwaj, M., Boubel, P., Boyce, M.M., Boyle, P.J., Brar, C., Burhanpurkar, M., Cassanelli, T., Chawla, P., Cliche, J.F., Cubranic, D., Deng, M., Denman, N., Dobbs, M., Fandino, M., Fonseca, E., Gaensler, B.M., Gilbert, A.J., Gill, A., Giri, U., Good, D.C., Halpern, M., Hanna, D.S., Hill, A.S., Hinshaw, G., Höfer, C., Josephy, A., Kaspi, V.M., Landecker, T.L., Lang, D.A., Lin, H.-H., Masui, K.W., Mckinven, R., Mena-Parra, J., Merryfield, M., Michilli, D., Milutinovic, N., Moatti, C., Naidu, A., Newburgh, L.B., Ng, C., Patel, C., Pen, U., Pinsonneault-Marotte, T., Pleunis, Z., Rafiei-Ravandi, M., Rahman, M., Ransom, S.M., Renard, A., Scholz, P., Shaw, J.R., Siegel, S.R., Smith, K.M., Stairs, I.H., Tendulkar, S.P., Tretyakov, I., Vanderlinde, K., Yadav, P.: A second source of repeating fast radio bursts. Nature 566, 235-238 (2019). https://doi.org/10.1038/s41586-018-0864-x

14. Corsi, A., Piro, L., Kuulkers, E., Amati, L., Antonelli, L.A., et al.: The puzzling case of GRB 990123: prompt emission and broad-band afterglow modeling. Astron. Astrophys. 438, 829-840 (2005)

15. Costa, E., Frontera, F., Heise, J., Feroci, M., J. in't Zand, Fiore, F., Cinti, M.N., Dal Fiume, D., Nicastro, L., Orlandini, M., Palazzi, E., Rapisarda, M., Zavattini, G., Jager, R., Parmar, A., Owens, A., Molendi, S., Cusumano, G., Maccarone, M.C., Giarrusso, S., Coletta, A., Antonelli, L.A., Giommi, P., 
Muller, J.M., Piro, L., Butler, R.C.: Discovery of an X-ray afterglow associated with the $\gamma$-ray burst of 28 February 1997. Nature 387, 783-785 (1997). https://doi.org/10.1038/42885

16. DeLaunay, J.J., Fox, D.B., Murase, K., Mészáros, P., Keivani, A., Messick, C., Mostafá, M.A., Oikonomou, F., Tešić, G., Turley, C.F.: Discovery of a transient gamma-ray counterpart to FRB 131104. ApJ 832, L1 (2016). https://doi.org/10.3847/2041-8205/832/1/L1

17. Drout, M.R., Chornock, R., Soderberg, A.M., Sanders, N.E., McKinnon, R., Rest, A., Foley, R.J., Milisavljevic, D., Margutti, R., Berger, E., Calkins, M., Fong, W., Gezari, S., Huber, M.E., Kankare, E., Kirshner, R.P., Leibler, C., Lunnan, R., Mattila, S., Marion, G.H., Narayan, G., Riess, A.G., Roth, K.C., Scolnic, D., Smartt, S.J., Tonry, J.L., Burgett, W.S., Chambers, K.C., Hodapp, K.W., Jedicke, R., Kaiser, N., Magnier, E.A., Metcalfe, N., Morgan, J.S., Price, P.A., Waters, C.: Rapidly evolving and luminous transients from Pan-STARRS1. ApJ 794, 23 (2014). https://doi.org/10.1088/0004-637X/794/1/23

18. Frontera, F., Virgilli, E., Guidorzi, C., Rosati, P., Diehl, R., Siegert, T., Fryer, C., Amati, L., Auricchio, N., Campana, R., Caroli, E., Fuschino, F., Labanti, C., Orlandini, M., Pian, E., Stephen, J., Del Sordo, S., Budtz-Jorgensen, C., Kuvvetli, I., Brandt, S., Curado da Silva, R., Laurent, P., Bozzo, E., Mazzali, P., Della Valle, M.: Understanding the origin of the positron annihilation line and the physics of supernova explosions. Exp. Astron. this issue (2021)

19. Fynbo, J.P.U., Watson, D., Thöne, C.C., Sollerman, J., Bloom, J.S., et al.: No supernovae associated with two long-duration $\gamma$-ray bursts. Nature 444, 1047-1049 (2006). https://doi.org/10.1038/nature05375

20. Ghisellini, G., Ghirlanda, G., Nava, L., Celotti, A.: GeV emission from gamma-ray bursts: a radiative fireball? MNRAS 403(2), 926-937 (2010). https://doi.org/10.1111/j.1365-2966.2009.16171.x

21. Glennie, A., Jonker, P.G., Fender, R.P., Nagayama, T., Pretorius, M.L.: Two fast X-ray transients in archival Chandra data. MNRAS 450(4), 3765-3770 (2015). https://doi.org/10.1093/mnras/stv801

22. Goldstein, A., Veres, P., Burns, E., Briggs, M.S., Hamburg, R., Kocevski, D., WilsonHodge, C.A., Preece, R.D., Poolakkil, S., et al.: An ordinary short gamma-ray burst with extraordinary implications: Fermi-GBM detection of GRB 170817A. ApJ 848, L14 (2017). https://doi.org/10.3847/2041-8213/aa8f41

23. Granot, J., Piran, T., Bromberg, O., Racusin, J.L., Daigne, F.: Gamma-ray bursts as sources of strong magnetic fields space science reviews. https://doi.org/10.1007/s11214-015-0191-6 (2015)

24. Guetta, D., Della Valle, M.: On the rates of gamma-ray bursts and type Ib/c Supernovae. ApJ 657, L73-L76 (2007). https://doi.org/10.1086/511417

25. Guidorzi, C., Margutti, R., Brout, D., Scolnic, D., Fong, W., Alexander, K.D., Cowperthwaite, P.S., Annis, J., Berger, E., et al.: Improved constraints on $\mathrm{H}_{0}$ from a combined analysis of gravitational-wave and electromagnetic emission from GW170817. ApJ 851, L36 (2017). https://doi.org/10.3847/2041-8213/aaa009

26. Guidorzi, C., Marongiu, M., Martone, R., Amati, L., Frontera, F., Nicastro, L., Orlandini, M., Margutti, R., Virgilli, E.: A search for gamma-ray prompt emission associated with the lorimer burst FRB 010724. ApJ 882(2), 100 (2019). https://doi.org/10.3847/1538-4357/ab3408

27. Irwin, J.A., Maksym, W.P., Sivakoff, G.R., Romanowsky, A.J., Lin, D., Speegle, T., Prado, I., Mildebrath, D., Strader, J., Liu, J., Miller, J.M.: Ultraluminous X-ray bursts in two ultracompact companions to nearby elliptical galaxies. Nature 538(7625), 356-358 (2016). https://doi.org/10.1038/nature19822

28. Jonker, P.G., Glennie, A., Heida, M., Maccarone, T., Hodgkin, S., Nelemans, G., Miller-Jones, J.C.A., Torres, M.A.P., Fender, R.: Discovery of a new kind of explosive X-ray transient near M86. ApJ 779(1), 14 (2013). https://doi.org/10.1088/0004-637X/779/1/14

29. Katz, J.I.: Fast radio bursts. Progr. Part. Nuclear Phys. 103, 1-18 (2018). https://doi.org/10.1016/j. ppnp.2018.07.001

30. Kouveliotou, C., Granot, J., Racusin, J.L., Bellm, E., Vianello, G., Oates, S., Fryer, C.L., Boggs, S.E., Christensen, F.E., Craig, W.W., Dermer, C.D., Gehrels, N., Hailey, C.J., Harrison, F.A., Melandri, A., McEnery, J.E., Mundell, C.G., Stern, D.K., Tagliaferri, G., Zhang, W.W.: NuSTAR observations of GRB 130427A establish a single component synchrotron afterglow origin for the late optical to multi-GeV emission. ApJ 779, L1 (2013). https://doi.org/10.1088/2041-8205/779/1/L1

31. Kulkarni, S.R., Frail, D.A., Wieringa, M.H., Ekers, R.D., Sadler, E.M., Wark, R.M., Higdon, J.L., Phinney, E.S., Bloom, J.S.: Radio emission from the unusual supernova 1998bw and its association with the $\gamma$-ray burst of 25 April 1998. Nature 395(6703), 663-669 (1998). https://doi.org/10.1038/27139 
32. Kumar, P., Zhang, B.: The physics of gamma-ray bursts and relativistic jets. Phys. Rep. 561, 1-109 (2015). https://doi.org/10.1016/j.physrep.2014.09.008

33. Lazzati, D.: X-ray polarization of gamma-ray bursts. 202. https://doi.org/10.1017/CBO9780511750809. 031 (2010)

34. Lazzati, D., Rossi, E., Ghisellini, G., Rees, M.J.: Compton drag as a mechanism for very high linear polarization in gamma-ray bursts. MNRAS 347, L1-L5 (2004). https://doi.org/10.1111/j.1365-2966. 2004.07387.x

35. Li, T.P., Xiong, S., Zhang, S., Lu, F., Song, L., Cao, X., Chang, Z., Chen, G., Chen, L., et al.: InsightHXMT observations of the first binary neutron star merger GW170817. Sci. China Phys. Mech. Astron. 61(3), 31011 (2018). https://doi.org/10.1007/s11433-017-9107-5

36. Liang, E., Zhang, B., Virgili, F., Dai, Z.G.: Low-luminosity gamma-ray bursts as a unique population: luminosity function, local rate, and beaming factor. ApJ 662, 1111-1118 (2007). https://doi.org/10.1086/517959

37. Lorimer, D.R., Bailes, M., McLaughlin, M.A., Narkevic, D.J., Crawford, F.: A bright millisecond radio burst of extragalactic origin. Science 318, 777 (2007). https://doi.org/10.1126/science.1147532

38. Luo, B., Brandt, W.N., Bauer, F.: A new highly variable X-ray source at z 1.5 in the Chandra deep field-south survey. The Astronomer's Telegram 6625, 1 (2014)

39. Lyutikov, M., Pariev, V.I., Blandford, R.D.: Polarization of prompt gamma-ray burst emission: evidence for electromagnetically dominated outflow. ApJ 597, 998-1009 (2003). https://doi.org/10. $1086 / 378497$

40. Maiorano, E., Masetti, N., Palazzi, E., Frontera, F., Grandi, P., et al.: The puzzling case of GRB 990123: multiwavelength afterglow study. Astron. Astrophys. 438, 821-827 (2005)

41. Margutti, R., Soderberg, A.M., Wieringa, M.H., Edwards, P.G., Chevalier, R.A., Morsony, B.J., Barniol Duran, R., Sironi, L., Zauderer, B.A., Milisavljevic, D., Kamble, A., Pian, E.: The signature of the central engine in the weakest relativistic explosions: GRB 100316D. ApJ 778, 18 (2013). https://doi.org/10.1088/0004-637X/778/1/18

42. Margutti, R., Guidorzi, C., Lazzati, D., Milisavljevic, D., Kamble, A., Laskar, T., Parrent, J., Gehrels, N.C., Soderberg, A.M.: Dust in the wind: the role of recent mass loss in long gamma-ray bursts. ApJ 805, 159 (2015). https://doi.org/10.1088/0004-637X/805/2/159

43. Margutti, R., Metzger, B.D., Chornock, R., Vurm, I., Roth, N., Grefenstette, B.W., Savchenko, V., Cartier, R., Steiner, J.F., Terreran, G., Margalit, B., Migliori, G., Milisavljevic, D., Alexander, K.D., Bietenholz, M., Blanchard, P.K., Bozzo, E., Brethauer, D., Chilingarian, I.V., Coppejans, D.L., Ducci, L., Ferrigno, C., Fong, W., Götz, D., Guidorzi, C., Hajela, A., Hurley, K., Kuulkers, E., Laurent, P., Mereghetti, S., Nicholl, M., Patnaude, D., Ubertini, P., Banovetz, J., Bartel, N., Berger, E., Coughlin, E.R., Eftekhari, T., Frederiks, D.D., Kozlova, A.V., Laskar, T., Svinkin, D.S., Drout, M.R., MacFadyen, A., Paterson, K.: An embedded x-ray source shines through the aspherical AT 2018cow: revealing the inner workings of the most luminous fast-evolving optical transients. ApJ 872(1), 18 (2019). https://doi.org/10.3847/1538-4357/aafa01

44. Martin-Carrillo, A., Hanlon, L., Topinka, M., LaCluyzé, A.P., Savchenko, V., Kann, D.A., Trotter, A.S., Covino, S., Krühler, T., Greiner, J.: GRB 120711A: an intense INTEGRAL burst with long-lasting soft $\gamma$-ray emission and a powerful optical flash. A\&A 567, A84 (2014). https://doi.org/10.1051/0004-6361/201220872

45. McConnell, M.L.: High energy polarimetry of prompt GRB emission. New A Rev. 76, 1-21 (2017). https://doi.org/10.1016/j.newar.2016.11.001

46. McGee, S., Sesana, A., Vecchio, A.: Linking gravitational waves and X-ray phenomena with joint LISA and Athena observations. Nat. Astron. 4, 26-31 (2020). https://doi.org/10.1038/s41550-0190969-7

47. Metzger, B.D., Margalit, B., Sironi, L.: Fast radio bursts as synchrotron maser emission from decelerating relativistic blast waves. MNRAS 485, $4091-4106$ (2019). https://doi.org/10.1093/mnras/stz700

48. Moretti, A., Margutti, R., Pasotti, F., Beardmore, A.P., Campana, S., Chincarini, G., Covino, S., Godet, O., Guidorzi, C., Osborne, J.P., Romano, P., Tagliaferri, G.: When GRB afterglows get softer, hard components come into play. A\&A 478(2), 409-417 (2008). https://doi.org/10.1051/0004-6361: 20078805

49. Morokuma-Matsui, K., Morokuma, T., Tominaga, N., Hatsukade, B., Hayashi, M., Tamura, Y., Matsuda, Y., Motogi, K., Niinuma, K., Konishi, M.: ALMA observations of molecular gas in the host galaxy of AT2018cow. ApJ 879(1), L13 (2019). https://doi.org/10.3847/2041-8213/ab2915 
50. Mundell, C.G., Steele, I.A., Smith, R.J., Kobayashi, S., Melandri, A., Guidorzi, C., Gomboc, A., Mottram, C.J., Clarke, D., Monfardini, A., Carter, D., Bersier, D.: Early optical polarization of a gamma-ray burst afterglow. Science 315, 1822 (2007). https://doi.org/10.1126/science.1138484

51. Mundell, C.G., Kopac, D., Arnold, D.M., Steele, I.A., Gomboc, A., Kobayashi, S., Harrison, R.M., Smith, R.J., Guidorzi, C., Virgili, F.J., Melandri, A., Japelj, J.: Highly polarized light from stable ordered magnetic fields in GRB 120308A. Nature 504, 119-121 (2013). https://doi.org/10.1038/nature12814

52. Murase, K., Kashiyama, K., Mészáros, P.: A burst in a wind bubble and the impact on baryonic ejecta: high-energy gamma-ray flashes and afterglows from fast radio bursts and pulsar-driven supernova remnants. MNRAS 461, 1498-1511 (2016). https://doi.org/10.1093/mnras/stw1328

53. Murase, K., Mészáros, P., Fox, D.B.: Fast radio bursts with extended gamma-ray emission? ApJ 836, L6 (2017). https://doi.org/10.3847/2041-8213/836/1/L6

54. Nakar, E., Sari, R.: Relativistic shock breakouts: a variety of gamma-ray flares: from low-luminosity gamma-ray bursts to type Ia Supernovae. ApJ 747, 88 (2012). https://doi.org/10.1088/0004-637X/ $747 / 2 / 88$

55. Nissanke, S., Holz, D.E., Hughes, S.A., Dalal, N., Sievers, J.L.: Exploring short gamma-ray bursts as gravitational-wave standard sirens. ApJ 725(1), 496-514 (2010). https://doi.org/10.1088/0004-637X/ 725/1/496

56. Pescalli, A., Ghirlanda, G., Salafia, O.S., Ghisellini, G., Nappo, F., Salvaterra, R.: Luminosity function and jet structure of gamma-ray burst. MNRAS 447, 1911-1921 (2015). https://doi.org/10.1093/mnras/ stu2482

57. Petroff, E., Hessels, J.W.T., Lorimer, D.R.: Fast radio bursts. Astron. Astrophys. Rev. 27(1), 4 (2019). ISSN 1432-0754. https://doi.org/10.1007/s00159-019-0116-6

58. Pian, E., Amati, L., Antonelli, L.A., Butler, R.C., Costa, E., Cusumano, G., Danziger, J., Feroci, M., Fiore, F., Frontera, F., Giommi, P., Masetti, N., Muller, J.M., Nicastro, L., Oosterbroek, T., Orlandini, M., Owens, A., Palazzi, E., Parmar, A., Piro, L., in’t Zand, J.JM., Castro-Tirado, A., Coletta, A., Dal Fiume, D., Del Sordo, S., Heise, J., Soffitta, P., Torroni, V.: BEPPOSAX observations of GRB 980425: detection of the prompt event and monitoring of the error box. ApJ 536(2), 778-787 (2000). https://doi.org/10.1086/308978

59. Punturo, M., Lück, H., Beker, M.: A third generation gravitational wave observatory: the Einstein telescope. In: Bassan, M. (ed.) Advanced interferometers and the search for gravitational waves, volume 404 of Astrophysics and Space Science Library, vol. 333 (2014). https://doi.org/10.1007/978-3-31903792-9_13

60. Pursiainen, M., Childress, M., Smith, M., Prajs, S., Sullivan, M., Davis, T.M., Foley, R.J., Asorey, J., Calcino, J., Carollo, D., Curtin, C., D’Andrea, C.B., Glazebrook, K., Gutierrez, C., Hinton, S.R., Hoormann, J.K., Inserra, C., Kessler, R., King, A., Kuehn, K., Lewis, G.F., Lidman, C., Macaulay, E., Möller, A., Nichol, R.C., Sako, M., Sommer, N.E., Swann, E., Tucker, B.E., Uddin, S.A., Wiseman, P., Zhang, B., Abbott, T.M.C., Abdalla, F.B., Allam, S., Annis, J., Avila, S., Brooks, D., BuckleyGeer, E., Burke, D.L., Carnero Rosell, A., Carrasco Kind, M., Carretero, J., Castander, F.J., Cunha, C.E., Davis, C., De Vicente, J., Diehl, H.T., Doel, P., Eifler, T.F., Flaugher, B., Fosalba, P., Frieman, J., García-Bellido, J., Gruen, D., Gruendl, R.A., Gutierrez, G., Hartley, W.G., Hollowood, D.L., Honscheid, K., James, D.J., Jeltema, T., Kuropatkin, N., Li, T.S., Lima, M., Maia, M.A.G., Martini, P., Menanteau, F., Ogando, R.L.C., Plazas, A.A., Roodman, A., Sanchez, E., Scarpine, V., Schindler, R., Smith, R.C., Soares-Santos, M., Sobreira, F., Suchyta, E., Swanson, M.E.C., G. Tarle, Tucker, D.L., Walker, A.R.: Rapidly evolving transients in the dark energy survey. MNRAS 481, 894-917 (2018). https://doi.org/10.1093/mnras/sty2309

61. Ravi, V., Catha, M., D’Addario, L., Djorgovski, S.G., Hallinan, G., Hobbs, R., Kocz, J., Kulkarni, S.R., Shi, J., Vedantham, H.K., Weinreb, S., Woody, D.P.: A fast radio burst localized to a massive galaxy. Nature 572(7769), 352-354 (2019). https://doi.org/10.1038/s41586-019-1389-7

62. Rest, A., Garnavich, P.M., Khatami, D., Kasen, D., Tucker, B.E., Shaya, E.J., Olling, R.P., Mushotzky, R., Zenteno, A., Margheim, S., Strampelli, G., James, D., Smith, R.C., Förster, F., Villar, V.A.: A fast-evolving luminous transient discovered by K2/Kepler. Nat. Astron. 2, 307-311 (2018). https://doi.org/10.1038/s41550-018-0423-2

63. Salafia, O.S., Ghisellini, G., Pescalli, A., Ghirlanda, G., Nappo, F.: Structure of gamma-ray burst jets: intrinsic versus apparent properties. MNRAS 450, 3549-3558 (2015). https://doi.org/10.1093/mnras/ stv766 
64. Salafia, O.S., Ghirlanda, G., Ascenzi, S., Ghisellini, G.: On-axis view of GRB 170817A. A\&A 628, A18 (2019). https://doi.org/10.1051/0004-6361/201935831

65. Scholz, P., Bogdanov, S., Hessels, J.W.T., Lynch, R.S., Spitler, L.G., Bassa, C.G., Bower, G.C., BurkeSpolaor, S., Butler, B.J., Chatterjee, S., Cordes, J.M., Gourdji, K., Kaspi, V.M., Law, C.J., Marcote, B., McLaughlin, M.A., Michilli, D., Paragi, Z., Ransom, S.M., Seymour, A., Tendulkar, S.P., Wharton, R.S.: Simultaneous X-Ray, gamma-ray, and radio observations of the repeating fast radio burst FRB 121102. ApJ 846, 80 (2017). https://doi.org/10.3847/1538-4357/aa8456

66. Sesana, A.: Prospects for multiband gravitational-wave astronomy after GW150914. Phys. Rev. Lett. 116(23), 231102 (2016). https://doi.org/10.1103/PhysRevLett.116.231102

67. Sironi, L., Spitkovsky, A.: Acceleration of particles at the termination shock of a relativistic striped wind. ApJ 741(1), 39 (2011). https://doi.org/10.1088/0004-637X/741/1/39

68. Smartt, S.J., Clark, P., Smith, K.W., McBrien, O., Maguire, K., O’Neil, D., Fulton, M., Magee, M., Prentice, S., Colin, C., Tonry, J., Denneau, L., Stalder, B., Heinze, A., Weiland, H., Flewelling, H., Rest, A.: ATLAS18qqn (AT2018cow) — a bright transient spatially coincident with CGCG 137-068 (60 Mpc). The Astronomer's Telegram 11727 (2018)

69. Soderberg, A.M., Kulkarni, S.R., Nakar, E., Berger, E., Cameron, P.B., et al.: Relativistic ejecta from X-ray flash XRF 060218 and the rate of cosmic explosions. Nature 442, 1014-1017 (2006). https://doi.org/10.1038/nature05087

70. Spitler, L.G., Scholz, P., Hessels, J.W.T., Bogdanov, S., Brazier, A., Camilo, F., Chatterjee, S., Cordes, J.M., Crawford, F., et al.: A repeating fast radio burst. Nature 531, 202-205 (2016). https://doi.org/10.1038/nature 17168

71. Steele, I.A., Mundell, C.G., Smith, R.J., Kobayashi, S., Guidorzi, C.: Ten per cent polarized optical emission from GRB090102. Nature 462, 767-769 (2009). https://doi.org/10.1038/nature08590

72. Tendulkar, S.P., Kaspi, V.M., Patel, C.: Radio nondetection of the SGR 1806-20 giant flare and implications for fast radio bursts. ApJ 827, 59 (2016). https://doi.org/10.3847/0004-637X/827/1/59

73. Tendulkar, S.P., Bassa, C.G., Cordes, J.M., Bower, G.C., Law, C.J., Chatterjee, S., Adams, E.A.K., Bogdanov, S., Burke-Spolaor, S., Butler, B.J., Demorest, P., Hessels, J.W.T., Kaspi, V.M., Lazio, T.J.W., Maddox, N., Marcote, B., McLaughlin, M.A., Paragi, Z., Ransom, S.M., Scholz, P., Seymour, A., Spitler, L.G., van Langevelde, H.J., Wharton, R.S.: The host galaxy and redshift of the repeating fast radio burst FRB 121102. ApJ 834, L7 (2017). https://doi.org/10.3847/2041-8213/834/2/L7

74. Toma, K., Sakamoto, T., Zhang, B., Hill, J.E., McConnell, M.L., Bloser, P.F., Yamazaki, R., Ioka, K., Nakamura, T.: Statistical properties of gamma-ray burst polarization. ApJ 698, 1042-1053 (2009). https://doi.org/10.1088/0004-637X/698/2/1042

75. Troja, E., Lipunov, V.M., Mundell, C.G., Butler, N.R., Watson, A.M., Kobayashi, S., Cenko, S.B., Marshall, F.E., Ricci, R., Fruchter, A., Wieringa, M.H., Gorbovskoy, E.S., Kornilov, V., Kutyrev, A., Lee, W.H., Toy, V., Tyurina, N.V., Budnev, N.M., Buckley, D.A.H., González, J., Gress, O., Horesh, A., Panasyuk, M.I., Prochaska, J.X., Ramirez-Ruiz, E., Rebolo Lopez, R., Richer, M.G., Roman-Zuniga, C., Serra-Ricart, M., Yurkov, V., Gehrels, N.: Significant and variable linear polarization during the prompt optical flash of GRB 160625B. Nature 547, 425-427 (2017). https://doi.org/10.1038/nature23289

76. Wei, J.-J., Wu, X.-F.: Precision test of the weak equivalence principle from gamma-ray burst polarization. Phys. Rev. D 99(10), 103012 (2019). https://doi.org/10.1103/PhysRevD.99.103012

77. Willingale, R., Mészáros, P.: Gamma-ray bursts and fast transients. Multi-wavelength observations and multi-messenger signals. Space Sci. Rev. 207, 63-86 (2017). https://doi.org/10.1007/s11214-0170366-4

78. Zhang, B.: A cosmic comb model of fast radio bursts. ApJ 836, L32 (2017). https://doi.org/10.3847/ 2041-8213/aa5ded

79. Zhang, B., Yan, H.: The internal-collision-induced magnetic reconnection and turbulence (ICMART) model of gamma-ray bursts. ApJ 726, 90 (2011). https://doi.org/10.1088/0004-637X/726/2/90

80. Zhang, S.-N., Kole, M., Bao, T.-W., Batsch, T., Bernasconi, T., Cadoux, F., Chai, J.-Y., Dai, Z.-G., Dong, Y.-W., Gauvin, N., Hajdas, W., Lan, M.-X., Li, H.-C., Li, L., Li, Z.-H., Liu, J.-T., Liu, X., Marcinkowski, R., Produit, N., Orsi, S., Pohl, M., Rybka, D., Shi, H.-L., Song, L.-M., Sun, J.-C., Szabelski, J., Tymieniecka, T., Wang, R.-J., Wang, Y.-H., Wen, X., Wu, B.-B., Wu, X., Wu, X.-F., Xiao, H.-L., Xiong, S.-L., Zhang, L.-Y., Zhang, L., Zhang, X.-F., Zhang, Y.-J., Zwolinska, A.: Detailed polarization measurements of the prompt emission of five gamma-ray bursts. Nat. Astron. 3, 258-264 (2019). https://doi.org/10.1038/s41550-018-0664-0 
Publisher's note Springer Nature remains neutral with regard to jurisdictional claims in published maps and institutional affiliations.

\section{Affiliations}

C. Guidorzi ${ }^{1,2,3}$ (1) F. Frontera ${ }^{1,2} \cdot$ G. Ghirlanda ${ }^{4,5} \cdot$ G. Stratta ${ }^{2} \cdot$ C. G. Mundell $\cdot$ E. Virgilli ${ }^{1}$. P. Rosati ${ }^{1,2,3} \cdot$ E. Caroli ${ }^{2} \cdot$ L. Amati ${ }^{2} \cdot$ E. Pian $^{2} \cdot$ S. Kobayashi ${ }^{7}$. G. Ghisellini ${ }^{4} \cdot$ C. Fryer ${ }^{8} \cdot$ M. Della Valle ${ }^{9,10,11} \cdot$ R. Margutti ${ }^{12} \cdot$ M. Marongiu $^{1,11}$. R. Martone ${ }^{1,11} \cdot$ R. Campana ${ }^{2} \cdot$ F. Fuschino ${ }^{2} \cdot$ C. Labanti ${ }^{2} \cdot$ M. Orlandini ${ }^{2}$. J. B. Stephen ${ }^{2} \cdot$ S. Brandt ${ }^{13} \cdot$ R. Curado da Silva ${ }^{14,15} \cdot$ P. Laurent ${ }^{16}$. R. Mochkovitch ${ }^{17}$. E. Bozzo ${ }^{18} \cdot$ R. Ciolfi ${ }^{19,20}$ - L. Burderi ${ }^{21} \cdot$ T. Di Salvo ${ }^{22}$

1 Department of Physics and Earth Science, University of Ferrara, Via Saragat 1, 44122 Ferrara, Italy

2 Observatory of Astrophysics and Space Sciences, National Institute of Astrophysics (INAF), Via Gobetti 101, 40129 Bologna, Italy

3 INFN - Sezione di Ferrara, via Saragat 1, 44122, Ferrara, Italy

4 INAF - Osservatorio Astronomico di Brera, via E. Bianchi 46, 23807 Merate, Italy

5 Dipartimento di Fisica G. Occhialini, Università di Milano Bicocca, Piazza della Scienza 3, 20126 Milano, Italy

6 Department of Physics, University of Bath, Claverton Down, Bath, BA2 7AY, UK

7 Astrophysics Research Institute, Liverpool John Moores University, Liverpool, L3 5RF, UK

8 Los Alamos National Laboratory, Los Alamos, NM 87545, USA

9 Capodimonte Astronomical Observatory, INAF-Napoli, Salita Moiariello 16, 80131 Napoli, Italy

10 Instituto de Astrofisica de Andalucia (IAA-CSIC), Glorieta de la Astronomıa, s/n, 18008, Granada, Spain

11 Internatinal Center for Relativistic Astrophysics, Piazzale della Repubblica 2, 65122 Pescara, Italy

12 Center for Interdisciplinary Exploration and Research in Astrophysics and Department of Physics and Astronomy, Northwestern University, 2145 Sheridan Road, Evanston, IL 60208-3112, USA

13 Technical University of Denmark, National Space Institute, Elektrovej, Denmark

14 LIP-Laboratório de Instrumentação e Física Experimental de Partículas, Lisboa, Portugal

15 Physics Department, University of Coimbra, Coimbra, Portugal

16 IRFU, CEA, Universite Paris-Saclay, 91191 Gif-sur-Yvette, France

17 Sorbonne Universite, CNRS, UMR 7095, Institut d'Astrophysique de Paris, 98 bis bd Arago, 75014 Paris, France

18 Department of Astronomy, University of Geneva, chemin d'Ecogia 16, 1290 Versoix, Switzerland

19 INAF, Osservatorio Astronomico di Padova, Vicolo dell'Osservatorio 5, 35122 Padova, Italy

20 INFN, Sezione di Padova, Via Francesco Marzolo 8, 35131 Padova, Italy

21 Dipartimento di Fisica, SP Monserrato-Sestu, Università degli Studi di Cagliari, km 0.7, 09042 Monserrato, Italy

22 Dipartimento di Fisica e Chimica, Università degli Studi di Palermo, Via Archirafi 36, 90123 Palermo, Italy 